\title{
WEAKLY BOUNDED SYSTEMS OF DIFFERENTIAL EQUATIONS
}

\author{
THOMAS G. HALLAM
}

1. Introduction. The differential equation

$$
x^{\prime}=f(t, x) \quad\left({ }^{\prime}=d / d t\right)
$$

will be considered with $f(t, x)$ continuous on $I \times R^{n}(I=\{t: 0 \leqq t<\infty\}$, $R^{n}$ is Euclidean $n$-space) and $f(t, 0)=0$ for $t$ in $I$. Furthermore, it will be assumed that (1) has a unique solution to the initial value problem and, thus, that the solutions depend continuously upon the initial data. The solution of (1) which passes through the point $\left(t_{0}, x_{0}\right)$ will be denoted by $x\left(t ; t_{0}, x_{0}\right)$. We will let $|x|$ designate any norm of the $n$-vector $x$ and $|A|$ designate any norm of the matrix $A$ which is consistent with the vector norm; that is, $|A x| \leqq|A||x|$.

An examination of the concepts of the stability $[1$, p. 56], $[3$, p. 31], $[9$, p. 26]), boundedness $[9$, p. 36], and practical stability $[3$, p. 121]; see also [7], leads to the following remark. Essentially, the above concepts are dependent upon two bounds: $\alpha$, the bound for the initial condition and $\beta$, the bound for the solution after the initial time. In order for (1) to possess the stability property, $\beta$ is preassigned and the existence of a suitable $\alpha$ must be demonstrated. For the boundedness definitions, the roles of $\alpha$ and $\beta$ are interchanged; that is, $\alpha$ is given and the bound on the solution $\beta$ must be determined. In the practical stability, both $\alpha$ and $\beta$ are preassigned.

The purpose of this note is to discuss some consequences of the following question: In the general theory of stability, what is the role of the existence of an $\alpha>0$ and a $\beta>0$ such that if $\left|x_{0}\right|<\alpha$, then $x\left(t ; t_{0}, x_{0}\right)$ is defined for $t \geqq t_{0}$ and $\left|x\left(t ; t_{0}, x_{0}\right)\right|<\beta$ for $t \geqq t_{0}$ ? Although this is a relatively weak property for the solutions of (1) to possess (when compared with the above stability concepts), in certain instances it is quite useful. The definitions used in this article will now be given.

Definition 1. Equation (1) will be called weakly bounded if for each $t_{0}$ in $I$ there exists an $\alpha=\alpha\left(t_{0}\right)>0$ and a $\beta=\beta\left(t_{0}\right)>0, \alpha \leqq \beta$, such that whenever $\left|x_{0}\right|<\alpha$ then $x\left(t ; t_{0}, x_{0}\right)$ is defined and $\left|x\left(t ; t_{0}, x_{0}\right)\right|<\beta$ for all $t \geqq t_{0}$. If, in addition, $\alpha$ and $\beta$ are constants (i.e. independent of $t_{0}$ ) then (1) will be called uniformly weakly bounded.

DEFINITION 2. Equation (1) will be called weakly contractive if there

Received by the editors May 8, 1967. 
exist constants $\alpha>0$ and $\beta>0, \alpha>\beta$, and a function $T=T\left(t_{0}\right)>0$ for all $t_{0}$ in $I$, such that if $\left|x_{0}\right|<\alpha$ then $x\left(t ; t_{0}, x_{0}\right)$ exists for all $t \geqq t_{0}$ and $\left|x\left(t ; t_{0}, x_{0}\right)\right|<\beta$ for all $t \geqq t_{0}+T$. If $T$ is independent of $t_{0}$ then (1) will be called uniformly weakly contractive.

It is clear that the equiboundedness of the solutions of (1), the stability of the zero solution of (1) or the practical stability of (1) implies that Equation (1) is weakly bounded. The following example shows that the weak boundedness of a system of differential equations is a different concept from boundedness and stability.

EXAmPLE 1. The differential equation

$$
x^{\prime}=x^{2}(x+1)^{2}(x-1)
$$

is (uniformly) weakly bounded for any $\alpha, 0<\alpha<1$, and $\beta, 1 \leqq \beta$. However, the solutions of (2) are not bounded, nor is the zero solution of (2) stable.

The next example is another illustration of weak boundedness and has applications in the asymptotic behavior of solutions of differential equations (see [2], [6]).

Example 2. Consider the differential equation

$$
x^{\prime}=a(t) x^{r}, \quad x \geqq 0, \quad t \in I,
$$

where $r>1, a(t)$ is nonnegative, continuous, and $\int^{\infty} a(t) d t<\infty$. The solutions of Equation (3) may be written as

$$
x(t)=\left[x\left(t_{0}\right)^{1-r}+(1-r) \int_{t_{0}}^{t} a(s) d s\right]^{1 /(1-r)} .
$$

The equation (3) is uniformly weakly bounded since, if $x\left(t_{0}\right)<\alpha$ $=[2 \gamma]^{1 /(1-r)}$, then $x(t)<\beta=\gamma^{1 /(1-r)}$, where $\gamma=(r-1) \int_{0}^{\infty} a(t) d t$.

REMARK. If equation (1) is weakly contractive, then equation (1) is weakly bounded. This result is a consequence of the assumption that (1) has a unique solution to the initial value problem.

2. Weak boundedness in linear systems. We will consider the linear homogeneous system of differential equations

$$
x^{\prime}=A(t) x
$$

where $A(t)$ is a continuous $n \times n$ matrix defined on $I$.

THEOREM 1. For Equation (4), the following conditions are equivalent:

(i) Equation (4) is (uniformly) weakly bounded.

(ii) The solutions of (4) are (uniformly) bounded.

(iii) The solutions of (4) are (uniformly) stable. 
Proof. If $X(t)$ is a fundamental matrix of solutions of (4), then $x\left(t ; t_{0}, x_{0}\right)=X(t) X^{-1}\left(t_{0}\right) x_{0}$. As a consequence of this representation, if (4) is weakly bounded for the bounds $\alpha$ (on the initial condition) and $\beta$ (on the solution), then for any solution $x\left(t ; t_{0}, x_{0}\right),\left|x\left(t ; t_{0}, x_{0}\right)\right|$ $\leqq \beta \alpha^{-1}\left|x_{0}\right|$. Using the fact that (uniform) boundedness of the solutions of (4) is equivalent to (uniform) equiboundedness [9, p. 37], we obtain (i) $\rightarrow$ (ii). The equivalence (ii) $\leftrightarrow$ (iii) is well known, [1, p. 54], $[9$, p. 34].

THEOREM 2. For the linear system (4), the following conditions are equivalent:

(i) Equation (4) is (uniformly) weakly contractive.

(ii) The solutions of (4) are (uniformly) ultimately bounded.

(iii) The solutions of (4) are (quasi-uniformly) asymptotically stable.

Proof. The proof will consist of showing (i) $\rightarrow$ (ii), since it is known that (ii) $\leftrightarrow$ (iii) $[9$, p. 45]. Let (4) be weakly contractive; suppose $\alpha, \beta$, and $T$ play the same role as in Definition 2. It will be shown that the solutions of (4) are ultimately bounded for the bound $\beta$.

Let $X(t)$ be a fundamental matrix of solutions of $(4)$; then

$$
\left|x\left(t ; t_{0}, x_{0}\right)\right|=\left|X(t) X^{-1}\left(t_{0}\right) x_{0}\right|<\beta,
$$

provided that $\left|x_{0}\right|<\alpha$ and $t \geqq t_{0}+T\left(t_{0}\right)$. Thus, for any $t_{0}$ in $I$, if $t \geqq t_{0}+T\left(t_{0}\right)$, then

$$
\left|X(t) X^{-1}\left(t_{0}\right)\right| \leqq \beta \alpha^{-1} .
$$

Suppose $x\left(t ; t_{0}, x_{0}\right)$ is any solution of $(4)$ with $\left|x_{0}\right|<\alpha_{0}$; then

$$
\left|x\left(t ; t_{0}, x_{0}\right)\right| \leqq\left|X(t) X^{-1}\left(t_{0}\right)\right|\left|x_{0}\right|<\beta \alpha^{-1} \alpha_{0}
$$

when $t \geqq t_{0}+T\left(t_{0}\right)$. Denoting by $t_{1}=t_{0}+T\left(t_{0}\right)$ and $x_{1}=x\left(t_{1} ; t_{0}, x_{0}\right)$, we observe that the point $\left(t_{1}, x_{1}\right)$ on the trajectory $x\left(t ; t_{0}, x_{0}\right)$ is such that $\left|x_{1}\right|<\beta \alpha^{-1} \alpha_{0}$. For each positive integer $k=1,2, \cdots$, we can continue in this manner to obtain points $\left(t_{k}, x_{k}\right)$ on the trajectory $x\left(t ; t_{0}, x_{0}\right)$ where $t_{k}=t_{k-1}+T\left(t_{k-1}\right), x_{k}=x\left(t_{k} ; t_{0}, x_{0}\right)$, and $\left|x_{k}\right|<\beta^{k} \alpha^{-k} \alpha_{0}$.

Since $\beta<\alpha$ there exists an integer $n$ such that $\beta^{n} \alpha^{-n} \alpha_{0}<\beta$; for such an integer $n$ we have

$$
\begin{aligned}
\left|x\left(t ; t_{0}, x_{0}\right)\right| & =\left|x\left(t ; t_{n-1}, x_{n-1}\right)\right| \\
& \leqq\left|X(t) X^{-1}\left(t_{n-1}\right)\right|\left|x_{n-1}\right| \\
& <\beta^{n} \alpha^{-n} \alpha_{0}<\beta,
\end{aligned}
$$

whenever $t \geqq t_{n-1}+T\left(t_{n-1}\right)$. Therefore the solutions of (4) are ultimately bounded for the bound $\beta$. 
The "uniform" part of the theorem follows from the above proof by observing that when $T$ is constant then the $t_{k}$ 's are given by $t_{k}=t_{0}+k T, k=1,2, \cdots, n-1$.

As a consequence of the above theorems, we observe that the Definitions 1 and 2 define distinct concepts, since examples are known [4], [8] for linear equations in which the corresponding types of boundedness of stability are different.

3. Weak boundedness in nonlinear systems. The next result, valid for periodic systems, is analogous to known results for boundedness [8] and stability [5]. The proof will be omitted because it proceeds in the classical manner.

THEOREM 3. Let $f(t+w, x)=f(t, x)$ for some $w \neq 0$ and for all $t$ and $x$, Equation (1) is weakly bounded if and only if (1) is uniformly weakly bounded.

Some sufficient conditions will be given which determine the weak boundedness of the solutions of a nonlinear system. Since Liapunov functions have been used to determine boundedness and stability of nonlinear systems, it seems logical that they would also apply in the instance of weak boundedness. This is indeed the case, as we shall see below.

As a scalar comparison differential equation, consider

$$
y^{\prime}=g(t, y)
$$

where $g(t, y)$ is continuous on $I \times R^{+}, R^{+}=\{y \mid 0 \leqq y<\infty\}$, and $g(t, 0) \equiv 0$ for $t \in I$. The Liapunov function $V(t, x)$ will be required to satisfy the following condition:

(6) $V(t, x)$ is a continuous function locally Lipschitz in $x$ which is defined for $t \in I$ and $0 \leqq|x|<\infty$ with $V(t, 0) \equiv 0$ for all $t \in I ; V(t, x)$ $\geqq a(|x|)$ where $a(r)$ is a continuous increasing function of $r$ for $r \geqq 0$ with $\lim _{r \rightarrow \infty} a(r)=\infty$. If

$$
V^{\prime}(t, x)=\limsup _{h \rightarrow 0^{+}}[V(t+h, x+h, f(t, x))-V(t, x)] / h,
$$

then $V^{\prime} \leqq g(t, V)$.

The proofs of the following theorems proceed in the classical manner and for this reason will be omitted.

THEOREM 4. Let condition (6) be satisfied. If the equation (5) is weakly bounded, then equation (1) is weakly bounded.

THEOREM 5. Let condition (6) be satisfied; furthermore, let $V(t, x)$ $\leqq b(|x|)$ where $b(r)$ is a continuous increasing function of $r$ for $r \geqq 0$. 
If equation (5) is uniformly weakly bounded, then equation (1) is uniformly weakly bounded.

There are analogous results for weakly contractive and uniform weakly contractive systems which correspond to Theorem 4 and 5 respectively. As an illustration of Theorem 5 we present

Example 3. For $|\cdot|$ denoting the Euclidean norm, let $f(t, x)$ satisfy the inequality

$$
|f(t, x)| \leqq a(t)|x|^{r}
$$

where $a(t)$ is continuous on $I, \int^{\infty} a(t) d t<\infty$, and $r>0$. As a Liapunov function, consider $V(x)=|x|^{2}$; then, $V$ satisfies the differential inequality

$$
V^{\prime} \leqq 2 a(t) V^{(r+1) / 2}
$$

If $r>0$, then the comparison differential equation associated with the above differential inequality

$$
y^{\prime}=2 a(t) y^{(r+1) / 2}
$$

is uniformly weakly bounded. In fact, for $r \leqq 1$, the solutions of (9) are uniformly bounded; and for $r>1$, from Example 2, we can conclude that (9) is uniformly weakly bounded. An application of Theorem 5 yields the result that equation (1) subject to condition (8) is uniformly weakly bounded.

\section{REFERENCES}

1. W. A. Coppel, Stability and asymptotic behavior of differential equations, Heath, Boston, Mass., 1965.

2. T. G. Hallam, Asymptotic behavior of the solutions of an nth order nonhomogeneous ordinary differential equation, Trans. Amer. Math. Soc. 122 (1966), 177-194.

3. J. P. LaSalle and S. Lefschetz, Stability by Liapunov's direct method with applications, Academic Press, New York, 1961.

4. J. L. Massera, On Liapounoff's conditions of stability, Ann. of Math. (2) 50 (1949), 705-721.

5. —_- Contributions to stability theory, Ann. of Math. 64 (1956), 182-206.

6. P. Waltman, On the asymptotic behavior of solutions of a nonlinear equation, Proc. Amer. Math. Soc. 15 (1964), 918-923.

7. L. Weiss and E. F. Infante, On the stability of systems defined over a finite time interval, Proc. Nat. Acad. Sci. U.S.A. 54 (1965), 44-48.

8. T. Yoshizawa, Liapunov's function and boundedness of solutions, Funkcial Ekvac. 2 (1958), 71-103.

9. - Stability theory by Liapunov's second method, Publ. Math. Soc. Japan, Tokyo, 1966.

Florida State University 